\title{
Malignant hyperthermia
}

\author{
Dong-Chan Kim
}

Department of Anesthesiology and Pain Medicine, Chonbuk National University Medical School, Jeonju, Korea

Malignant hyperthermia ( $\mathrm{MH})$ is an uncommon, life-threatening pharmacogenetic disorder of the skeletal muscle. It presents as a hypermetabolic response in susceptible individuals to potent volatile anesthetics with/without depolarizing muscle relaxants; in rare cases, to stress from exertion or heat stress. Susceptibility to malignant hyperthermia (MHS) is inherited as an autosomally dominant trait with variable expression and incomplete penetrance. It is known that the pathophysiology of $\mathrm{MH}$ is related to an uncontrolled rise of myoplasmic calcium, which activates biochemical processes resulting in hypermetabolism of the skeletal muscle. In most cases, defects in the ryanodine receptor are responsible for the functional changes of calcium regulation in $\mathrm{MH}$, and more than 300 mutations have been identified in the RYR1 gene, located on chromosome 19q13.1. The classic signs of MH include increase of end-tidal carbon dioxide, tachycardia, skeletal muscle rigidity, tachycardia, hyperthermia and acidosis. Up to now, muscle contracture test is regarded as the gold standard for the diagnosis of MHS though molecular genetic test is used, on a limited basis so far to diagnose MHS. The mortality of MH is dramatically decreased from $70-80 \%$ to less than $5 \%$, due to an introduction of dantrolene sodium for treatment of $\mathrm{MH}$, early detection of $\mathrm{MH}$ episode using capnography, and the introduction of diagnostic testing for MHS. This review summarizes the clinically essential and important knowledge of $\mathrm{MH}$, and presents new developments in the field. (Korean J Anesthesiol 2012; 63: 391-401)

Key Words: Diagnosis, Epidemiology, Malignant hyperthermia, Pathophysiology, Treatment.

\section{Introduction}

Malignant hyperthermia ( $\mathrm{MH}$ ) is an inherited, pharmacogenetic skeletal muscle disorder involving the dysregulated myoplasmic $\mathrm{Ca}^{2+}$, hypercontracture, and hypermetabolism in response to an exposure to potent volatile anesthetics with/ without depolarizing muscle relaxants, and can be triggered by exertional or heat stress without pharmacologic triggering agents in rare cases $[1,2]$. Susceptibility to malignant hyperthermia (MHS) is inherited as an autosomally dominant trait with variable expression and incomplete penetrance [1]. As almost all individuals with MHS appear normal without any pathologic signs, it is impossible to diagnose susceptibility without either the exposure to triggering agents or by specific

Received: September 4, 2012. Accepted: September 10, 2012.

Corresponding author: Dong-Chan Kim, M.D., Ph.D., Department of Anesthesiology and Pain Medicine, Chonbuk National University Medical School, 634-18, Keumam-dong, Deokjin-gu, Jeonju 561-712, Korea. Tel: 82-63-250-1251, Fax: 82-63-250-1240, E-mail: dckim@chonbuk.ac.kr (c) This is an open-access article distributed under the terms of the Creative Commons Attribution Non-Commercial License (http:// creativecommons.org/licenses/by-nc/3.0/), which permits unrestricted non-commercial use, distribution, and reproduction in any medium, provided the original work is properly cited. 
diagnostic testing.

Although the estimated genetic prevalence of MHS is reported as up to $1: 2,000-3,000$ in individuals [3-5], $\mathrm{MH}$ episodes are very rare and modern anesthetic techniques, such as increasing use of non-triggering intravenous anesthetics and avoidance of succinylcholine are more likely to make it even rarer, leading to the potential risk of reduced awareness of anesthesiologists for $\mathrm{MH}$.

When MH was first recognized as a complication of anesthesia, the mortality rate was $70-80 \%$ [6]. Nowadays, the mortality rate is estimated to be less than $5 \%$, with early detection of $\mathrm{MH}$ episode, using capnography, prompt use of the drug dantrolene, and the introduction of diagnostic testing [7]. Even though the mortality rates of $\mathrm{MH}$ are low, according to a recent study by Larach et al. [8], the morbidity rate of $\mathrm{MH}$ is $34.8 \%$. This high morbidity rate emphasizes the need for continuing education of anesthesiologists on the most effective way to diagnose and treat $\mathrm{MH}$.

The purpose of this review is to summarize the clinically essential and important knowledge of $\mathrm{MH}$ and presents new developments in the field.

\section{Epidemiology}

Although the exact incidence of malignant hyperthermia is unknown, the incidence during general anesthesia is estimated to range from $1: 5,000$ to $1: 50,000-100,000$ in individuals $[7,9]$. However, the true number of individuals with MHS is likely to be much greater, as individuals with MHS may undergo anesthesia uneventfully several times before a clinical episode occurs and many cases of MH may go undetected because many individuals with MHS are never anesthetized, have short durations of exposure, or have mild, uncomplicated presentations that are never diagnosed. Generally, the estimated prevalence of MHS is thought to be approximately 1 in 50,000 or less. However, according to the results of recent molecular genetic studies, the estimated genetic prevalence may be up to $1: 2,000-3,000$ because MHS is inherited as an autosomally dominant trait [3-5].

$\mathrm{MH}$ has been reported in every country, and all races are susceptible [7]. MH occurs more frequently in males than females $[7,8]$, and more commonly in children and young adults with the mean age of 18.3 years [10]. Although reported in the newborn, the earliest reaction confirmed by testing is six months of age [10]. Although the exact cause for male predominance of $\mathrm{MH}$ is unclear, suggested hypothesis includes differences in ionic channels for muscle contraction influenced by either sex hormones or native differences among genders, higher incidences false-positive diagnosis among males, as well as a difference in genetic inheritance [11-13]. In a prevalence and clinical outcome study from New York State from 2001 to 2005, the estimated prevalence of $\mathrm{MH}$ for males was 2.5-4.5 times the rate for females, and the median age at presentation was 22 years with $45 \%$ of $\mathrm{MH}$ cases occurring in those who were 19 years or younger [14].

The mortality rates of $\mathrm{MH}$ dramatically decreased from $70-$ $80 \%$ to $10 \%$ after an introduction of dantrolene sodium for the treatment of $\mathrm{MH}$, and recent mortality is estimated to be less than $5 \%$ with early detection of $\mathrm{MH}$ episode using capnography, prompt use of the drug dantrolene, and the introduction of diagnostic testing [6,7]. Although the mortality rates of $\mathrm{MH}$ are low, the morbidity rate of $\mathrm{MH}$ is much higher. According to the recent study of Larach et al.[8], the morbidity rate of $\mathrm{MH}$ is $34.8 \%$ and frequently reported complications are changes in the consciousness level/coma (9.8\%), cardiac dysfunction (9.4\%), pulmonary edema (8.4\%), renal dysfunction (97.3\%), disseminated intravascular coagulation (7.2\%), and hepatic dysfunction (5.6\%). This high morbidity rate emphasizes the need for continuing education of anesthesiologists on the most effective way to diagnose and treat $\mathrm{MH}$.

\section{Pathophysiology}

Malignant hyperthermia, a dominantly inherited pharmacogenetic skeletal muscle disorder characterized by skeletal muscle hypermetabolism, occurs in susceptible individuals following exposure to potent halogenated anesthetics or depolarizing muscle relaxants during anesthesia [7]. Although the exact mechanism by which triggering agents initiate $\mathrm{MH}$ has not been fully elucidated yet, numerous experimental evidences from various sources clearly indicate that underlying causes of this hypermetabolism are related to an uncontrolled release of calcium from skeletal muscle sarcoplasmic reticulum (SR) [1,2,15-18].

During excitation-contraction coupling, muscle membrane depolarization induces a conformational change in the dihydropyridine-sensitive L-type voltage-dependent calcium channel (DHPR). This conformational change leads to activation of the ryanodine receptor type 1 (RYR1) and the rapid release of $\mathrm{Ca}^{2+}$ from its stores in the SR. Released $\mathrm{Ca}^{2+}$ binds to troponin $\mathrm{C}$, causing movement of tropomyosin away from the myosin-binding sites on the thin filament and triggering muscle contraction. Muscle contraction is terminated when $\mathrm{Ca}^{2+}$ is actively pumped back into the SR by the ATP-dependent calcium pump. This process is the fundamental excitationcontraction coupling (ECC) needed for normal skeletal muscle function $[19,20]$.

In the early phase of $\mathrm{MH}$, the muscle cells attempt to restore homeostasis by sequestering calcium through increasing in aerobic and anaerobic metabolism [7]. However, in malignant 
hyperthermia susceptible muscle, the uncontrolled release of calcium and the rise in myoplasmic calcium caused by the triggering agents overpowers the cellular capacity to reestablish homeostasis. This pathologically enhanced myoplasmic calcium rise eventually reaches the threshold levels for myofibrillar contraction, and results in sustained muscle contraction. The sustained muscle contraction produces a rapid depletion of adenosine triphosphate (ATP) with a concomitant increase in glucose metabolism, oxygen consumption, carbon dioxide production, and heat production. ATP stores become depleted, which progressively lead to the failure of membrane integrity with leakage of muscle cell contents (including electrolytes, myoglobin and various other sarcoplasmic proteins, like CK into the circulation [7].

The cause of dysfunctional calcium regulation in skeletal muscle with MHS lies, in most cases, in a defective RYR1 in SR, which is the footplate protein seated between the DHPR and SR [21-23]. Major defects in these two receptors (RYR1 and DHPR), as well as in other proteins, such as, triadin and FK 506 binding protein involved in the myoplasmic calcium regulation, are responsible for the functional changes of calcium regulation in $\mathrm{MH}$. It is widely accepted that the gene of RYRl (RYRI) is the major, though not exclusive, site for mutations associated with MHS $[24,25]$. Approximately $70 \%$ of families with MHS carry mutations in RYR1 [26-28].

Although mutations in RYR1 gene are undoubtedly important in the pathophysiology of MH, it is clear that not all families exhibit linkage to this gene. Other potential genetic loci have been identified, such as mutations in CACNAIS encodes the $\alpha 1$ subunit of DHPR $[29,30]$.

\section{Clinical Presentation and Diagnosis}

The diagnosis of MH is based on clinical presentations or laboratory testing. However, the clinical presentations and first signs of $\mathrm{MH}$ episode are highly variable, especially when some individuals exhibit only one or a few symptoms of varying intensity; it is not easy to diagnose $\mathrm{MH}$ with confidence $[31,32]$. Although the underlying causes for the variability of the clinical presentation are poorly understood, different potency, concentration and duration of exposure to the triggering inhalation anesthetics, as well as additional factors, such as temperature, age and genetic variability, may be related with the heterogeneous clinical presentations $[31,33,34]$. The variability in the order and time of onset of signs often makes the clinical diagnosis rather difficult $[7,35,36]$.

The characteristic clinical signs of $\mathrm{MH}$, during general anesthesia, are tachycardia, unexplained elevation of end-tidal carbon dioxide $\left(\mathrm{ET}_{\mathrm{CO} 2}\right)$ concentration, muscle rigidity, acidosis, hyperthermia, and evidence of rhabdomyolysis. The most frequent and the earliest sign of $\mathrm{MH}$ is unexplained sinus tachycardia, together with unexplained elevation of end-tidal carbon dioxide. In a clinical analysis study of MH cases reported to the North American Malignant Hyperthermia Registry from 1987 to 2006 [8], frequent first signs of $\mathrm{MH}$ include hypercapnia (92\%), sinus tachycardia (73\%), and masseter muscle rigidity (27\%). In $63.5 \%$ of cases, temperature abnormality (median maximum, $39.1^{\circ} \mathrm{C}$ ) was the first to third sign. Whereas $78.6 \%$ presented both muscular abnormalities and respiratory acidosis, only $26.0 \%$ had metabolic acidosis.

The unexplained elevation of end-tidal carbon dioxide may be the earliest and the most common sign of MH episode in the intubated and mechanically ventilated patient [37]. Especially, when succinylcholine is used as the relaxing agent, abrupt rise of end-tidal carbon dioxide can be observed [38]. However, with a decline in the use of succinylcholine, a more gradual rise in $\mathrm{CO}_{2}$, rather than an abrupt rise are often noted, and it is possible to mask this rise by increasing the minute ventilation [39].

Sinus tachycardia is observed in almost all patients as one of the early signs of MH episode, and may be misinterpreted as an inadequate anesthetic depth.

Although the severe, rapidly developing hyperthermia is considered as a hallmark of $\mathrm{MH}$ from when malignant hyperthermia is described firstly in 1960 to nowadays, increasing body temperature is a relatively late sign of $\mathrm{MH}$ and is even absent sometimes $[40,41]$. Therefore, one should not wait for this sign to appear before making the diagnosis. When hyperthermia occurs, the rate of temperature increase may be as rapid as $1-2^{\circ} \mathrm{C}$ every five minutes, up to greater than $44^{\circ} \mathrm{C}$, and leads to a marked increase in oxygen consumption, carbon dioxide production, widespread vital organ dysfunction, and disseminated intravascular coagulation (DIC)[42]. The rate of temperature elevation is much more of a differential diagnostic and prognostic value than peak temperature, though higher maximum temperatures significantly increase the likelihood of $\mathrm{MH}$ complications $[2,8]$. For patient safety, a monitoring of core temperature is essentially recommended in all anesthetics expected to last $\geq 30$ minutes $[8,43]$.

Generalized muscular rigidity is a definitive sign of $\mathrm{MH}$ and reflects the $\mathrm{Ca}^{2+}$-dependent activation of muscle contraction accompanying activation of both aerobic and anaerobic metabolism. About $50-80 \%$ of the patients present generalized muscular rigidity [2]. If $\mathrm{MH}$ is suspected, peripheral muscular rigidity should be checked [41].

The change in arterial pressure is not usually marked in the early phase of $\mathrm{MH}$. As $\mathrm{MH}$ episode progresses, late clinical signs, such as cyanosis, cardiac arrhythmias, mixed respiratory and metabolic acidosis, and various electrolyte imbalances arise.

Rhabdomyolysis is another clinical sign related to the dest- 
Table 1. Criteria Used in the Clinical Grading Scale for Malignant Hyperthermia (Adapted from Larach et al. [46] and Rosenberg et al. [7])

\begin{tabular}{ll}
\hline Clinical manifestation & \multicolumn{1}{c}{ Indicator } \\
\hline Respiratory acidosis & End-tidal $\mathrm{CO}_{2}>55 \mathrm{mmHg}$ or $\mathrm{PaCO}_{2}>60 \mathrm{mmHg}(15)$ \\
Cardiac involvement & Unexplained sinus tachycardia, ventricular tachycardia or ventricular fibrillation (3) \\
Metabolic acidosis & Base deficit $>-8 \mathrm{mEq} / \mathrm{L}$ or $\mathrm{pH}<7.25(10)$ \\
Muscle rigidity & Generalized rigidity or severe masseter muscle rigidity (15) \\
Muscle breakdown & Serum creatine kinase concentration $>20,000 \mathrm{IU}(15) ;$ cola colored urine (10); excess myoglobin \\
& in urine or serum (5); plasma $\left[\mathrm{K}^{+}\right]>6 \mathrm{mEq} / \mathrm{L} \mathrm{(3)}$ \\
Temperature increase & Rapidly increasing temperature; $>38.8^{\circ} \mathrm{C}(15)$ \\
Other & Rapid reversal of $\mathrm{MH}$ signs with dantrolene (5) \\
Family history & Consistent with autosomal dominant inheritance (15) \\
\hline
\end{tabular}

Clinical manifestations (except family history) are in order of relative importance. Indicator means clinical of laboratory signs occurring during or shortly after general anesthesia in the untreated individual. The number in a parenthesis means score.

Table 2. Interpreting the Raw Score: MH Rank and Qualitative Likelihood (Adapted from Larach et al. [46])

\begin{tabular}{ccl}
\hline Raw score range & MH rank & Description of likelihood \\
\hline 0 & 1 & Almost never \\
$3-9$ & 2 & Unlikely \\
$10-19$ & 3 & Somewhat less than likely \\
$20-34$ & 4 & Somewhat greater than likely \\
$35-49$ & 5 & Very likely \\
$>50$ & 6 & Almost certain \\
\hline
\end{tabular}

ruction of skeletal muscle and is manifested by brown or colacolored urine. Acute renal failure may occur due to myoglobin precipitation in the renal tubules and close observation and laboratory monitoring for myoglobin should be instituted [44].

Disseminated intravascular coagulation (DIC) is a poor prognostic sign in $\mathrm{MH}$. Larach et al. [45] found that DIC was associated with a 50 -fold increased likelihood of cardiac arrest and an 89-fold likelihood of death.

Numerous conditions may resemble MH during anesthesia that present unspecific signs, such as tachycardia, hypercapnia, and hyperthermia, which may be confused with $\mathrm{MH}$. These include surgical stress, inadequate anesthetic depth, sepsis, thyroid storm, pheochromocytoma, iatrogenic overheating, malfunction of anesthetic equipment, and etc. [7,41].

Larach et al. [46] had developed a clinical grading scale in order to assist in a clinical diagnosis of MH in 1994. The clinical grading scale is a scoring system that gives differential score to the various clinical manifestations of MH episode, such as muscle rigidity, muscle breakdown, respiratory acidosis, metabolic acidosis, temperature increase, cardiac involvement and family history. The scores are summed and categorized into six different groups to assess the likelihood of an intraoperative event representing an $\mathrm{MH}$ episode. The elements of the scoring system and interpretation are given in Table 1 and 2 . This scoring system allows us some guidance in deciding whether a patient may be suffering from $\mathrm{MH}$ or not, without the use of specialized diagnostic testing. However, if data are partly or totally unavailable, which is common, the clinical grading scale lacks sensitivity to diagnose $\mathrm{MH}$ since evaluation using this scale depends on the availability of data $[47,48]$.

\section{Laboratory Diagnostic Tests for MHS}

As almost all individuals with MHS are asymptomatic during daily life, specific testing is needed to confirm or exclude MHS. In probands who have survived from a suspected $\mathrm{MH}$ episode, the confirmation of MHS diagnosis is very important because other family members may be affected by this dominantly inherited disorder. Also, the exclusion of MHS diagnosis in relatives of proband is equally important because $\mathrm{MH}$ is almost fatal if not rapidly diagnosed and treated. It would be a significant improvement for anesthesia risk management if we could reliably predict or confirm MHS [31,49].

The standard test for MHS diagnosis is the muscle contracture test, and recently, molecular genetic test is introduced.

\section{The muscle contracture test}

The most widely used and the most sensitive method for determining whether an individual is susceptible to $\mathrm{MH}$ is the muscle contracture test [32]. The muscle contracture test for diagnosis of MHS that involves exposing living muscle fiber bundles to halothane, and separately to caffeine according to a strict protocol, was developed based on the observations of Kalow et al. [50] and Ellis et al. [51] in 1970s.

After the processes of standardization (e.g., which muscle to biopsy, exposure regimens and diagnostic thresholds) to develop an accurate diagnostic test for MHS, this has been the "gold standard" for diagnosis of MHS. These processes of standardization led to the formation of two slightly different protocols, a European version (IVCT; the in vitro contracture test) by the European Malignant Hyperthermia Group (EMHG) and a North American version (CHCT; the caffeinehalothane contracture test) by the North American Malignant 
Hyperthermia Group (NAMHG) [52,53].

While similarities exist in performing and interpreting the results of tests, such as the criteria for muscle sampling, muscle biopsy extraction to testing time, and muscle viability assessments, there are significant differences between the two protocols $[52,53]$. When muscle is acquired anatomically, the NAMHG protocol may use any muscle site; whereas, the EMHG protocol limits muscle specimens from the vastus lateralis. The NAMHG protocol uses exposure to a single concentration of halothane (3\%) with a range of diagnostic thresholds $(0.2-0.7 \mathrm{~g})$, while the EMHG protocol uses incremental exposure to halothane $(0.5,1$, $2 \%$ ) with a uniform diagnostic threshold ( $\geq 0.2 \mathrm{~g}$ ). The NAMHG protocol considers a positive CHCT result if any one of the three bundles exposed to halothane or the three bundles exposed to caffeine exhibit a positive response (contracture of $\geq 0.7 \mathrm{~g}$ to $3 \%$ halothane or contracture of $\geq 0.3 \mathrm{~g}$ to $2.0 \mathrm{mMl} / \mathrm{L}$ caffeine), then the individual tested is judged to be MH susceptible (MHS); otherwise, he or she is considered normal (MHN) $[54,55]$. In contrast, the EMHG protocol requires a positive response (contracture of $\geq 0.2 \mathrm{~g}$ to $\leq 2 \%$ halothane) in one of the two bundles tested with halothane and a positive response (contracture of $\geq 0.2 \mathrm{~g}$ to $\leq 2.0 \mathrm{mmol} / \mathrm{L}$ caffeine) in one of the two bundles with caffeine to be diagnosed as MH susceptible (MHS) [56]. If the results of the halothane and caffeine tests are negative, then the individual is diagnosed as normal (MHN). If a positive response occurs only to halothane or to caffeine, the test result is labeled as $\mathrm{MH}$ equivocal (MHE), although the individual is regarded clinically as MHS. This individual is further subdivided into MHE (h) and MHE (c), depending on whether the positive response was to halothane or to caffeine, respectively [52]. Currently, North American testing centers use a lower threshold for MHS, referred to as a clinical diagnosis (contracture $\geq 0.5$ g to $3 \%$ halothane, and a contracture $\geq 0.3$ $\mathrm{g}$ to $2 \mathrm{mM}$ caffeine), to include as many susceptible patients as possible with the possibility of misdiagnosing a patient as MHS when they might be MHN. When individuals are going to be used in genetic studies, a higher threshold (halothane contracture $\geq 0.7 \mathrm{~g}$ ) for diagnosis of MHS is used. As such, only patients truly susceptible will be included in research [57].

Like any tests used in clinical medicine, muscle contracture testing has limits on its sensitivity and specificity. Because $\mathrm{MH}$ episode is potentially fatal, the thresholds for diagnostic testing require a high degree of sensitivity and an acceptable degree of specificity. This means that anesthesiologists are willing to accept false-positive responses to avoid false-negative ones because the consequences of a false-negative diagnosis might be disastrous. Consequently, the diagnostic thresholds were established to minimize the possibility of false-negative results.

The EMHG protocol may reduce the possibility of false positive and negative results, when compared to that of the
NAMHG protocol; however, obtained results were similar, overall [58]. Despite the use of low cutoff for interpreting and risking higher false positive in the EMHG protocol, the IVCT has a $99 \%$ of sensitivity and a $93.6 \%$ of specificity, while the NAMHG protocol has a sensitivity and a specificity of $97-98 \%$ and $78-80 \%$, respectively $[55,56]$. Thus, there are a $1 \%$ chance of misdiagnosing a $\mathrm{MH}$-susceptible individual as $\mathrm{MHN}$, and a $6 \%$ chance of misdiagnosing a individual without susceptibility as MHS in the EMHG protocol; whereas there are a $2-3 \%$ chance that test will incorrectly mislabel a MH-susceptible individual as MHN, and a $20 \%$ chance that a individual without susceptibility will be labeled as MHS in the NAMHG protocol.

Although the decision to proceed with muscle contracture test depends on numerous factors, including the features of the suspected MH event, family history, patients' willingness, etc, in general, muscle contracture test should be performed on individuals with a clinical history of $\mathrm{MH}$ or close relatives of known MH susceptible individuals to exclude MHS [32].

Even though muscle contracture test still regarded as the gold standard for the diagnosis of MHS, this test is invasive, expensive, and can be performed only at specialized centers. Therefore, the search for a less invasive method than IVCT/ CHCT to diagnose MHS has been ongoing for many years, and molecular genetic testing based on advances in molecular genetics and cellular physiology has been introduced as an attractive alternative [32,59].

\section{The molecular genetic testing}

Although molecular genetic testing for MHS has been introduced relatively recently, it is used as a diagnostic test for MHS, in limited capacity, in Europe and North America [60-62].

Molecular genetic testing was first suggested as a method of MHS diagnosis in 1990, when a mutation within RYR1 encoding the skeletal muscle calcium release channel was identified [63]. Since then, it was identified that about $70 \%$ of MHS have been linked to $R Y R 1$ with over 300 mutations associated with MHS $[28,61,64]$. With the identification of causative mutations in RYR1, and the rapid developments in molecular genetics technology, molecular genetic testing is expected to be a screening test for MHS diagnosis. However, it is not realized at this time because of the metabolic complexity, discordance between phenotype and genotype, and genetic heterogeneity of MHS [65-68].

At present, despite more than $300 \mathrm{MHS}$ associated mutations in $R Y R 1$ have been identified, only 30 functionally confirmed causative point mutations have been approved as diagnostic mutations for MHS by EMHG, and 29 have been formally accepted as causative mutations by the MAMHG [61]. This means that these only 30 causative mutations can be used for screening of MHS diagnosis. The most important limitation of 
screening of MHS, using molecular genetic test at this time, is that the absence of causative RYR1 mutations itself cannot rule out MHS without muscle contracture test. Therefore, molecular genetic test for MHS diagnosis should always be used in selected and genetically characterized families, as well as under the guidelines for molecular genetic test recommended by the EMHG [60,62].

When MHS is confirmed in a proband or appropriate family member by muscle contracture test and a causative mutation is identified, then molecular genetic test becomes valuable for other members of the family, as other members of the family can be diagnosed as MHS by relatively a simple test for the presence of the same familial mutation, without muscle contracture test. Mutation-positive members would be regarded as MHS without muscle contracture test. However, individuals in whom the familial mutation was not identified must undergo muscle contracture test, in order to confirm or exclude MHS [60,62].

Molecular genetic test for MHS diagnosis has some limitations, including the relatively low sensitivity (25\%) and the considerable amount of interindividual and intraindividual variability in phenotypic expression in individuals with MHS $[32,69]$. Ongoing investigations will unveil further causative mutations, and thereby improve the usefulness and efficacy of molecular genetic testing in the future.

\section{Management of MH Episode}

Early recognition of the early signs of $\mathrm{MH}$ episode and immediate starting of appropriate treatment is essential for successful treatment of $\mathrm{MH}$ as the prognosis is poorer if late signs appear. Although the early signs of $\mathrm{MH}$ episode are nonspecific, anesthesiologists should be familiar with the nature of the condition and clinical signs of $\mathrm{MH}$ episode, and maintain awareness during general anesthesia in order to diagnose rapidly. Table 3 lists the clinical signs associated with $\mathrm{MH}$. Because the clinical presentation of $\mathrm{MH}$ episode highly varies, treatment should be modified accordingly [70].

Once $\mathrm{MH}$ episode is suspected, all triggering agents should be discontinued immediately and the vaporizer for the volatile anesthetic should be removed from the anesthesia machine. If the surgical procedure must continue after discontinuation of triggering agents, anesthesia should be maintained using non-triggering agents, including opioids, sedatives, and nondepolarizing muscle relaxants.

It is imperative to call additional experienced help immediately because the coordinated team approach will lead to the most efficient implementation of the recommended interventions.

The patient should be hyperventilated with $100 \%$ oxygen ( $>10 \mathrm{~L} / \mathrm{min}$ ) through a non-rebreathing circuit at $2-3$ times the normal minute ventilation to correct hypoxemia and respiratory acidosis.

The only specific drug to manage $\mathrm{MH}$ is dantrolene sodium, a specific ryanodine receptor antagonist that inhibits the pathologically increased calcium release from the sarcoplasmic reticulum in the affected individuals $[71,72]$. The recommended initial dosage is $2.5 \mathrm{mg} / \mathrm{kg}$ administered intravenously every 5 minutes, up to the total of $10 \mathrm{mg} / \mathrm{kg}$ until the hypermetabolism is

Table 3. Clinical Signs Associated with MH (Adapted from Glagn et al. [70])

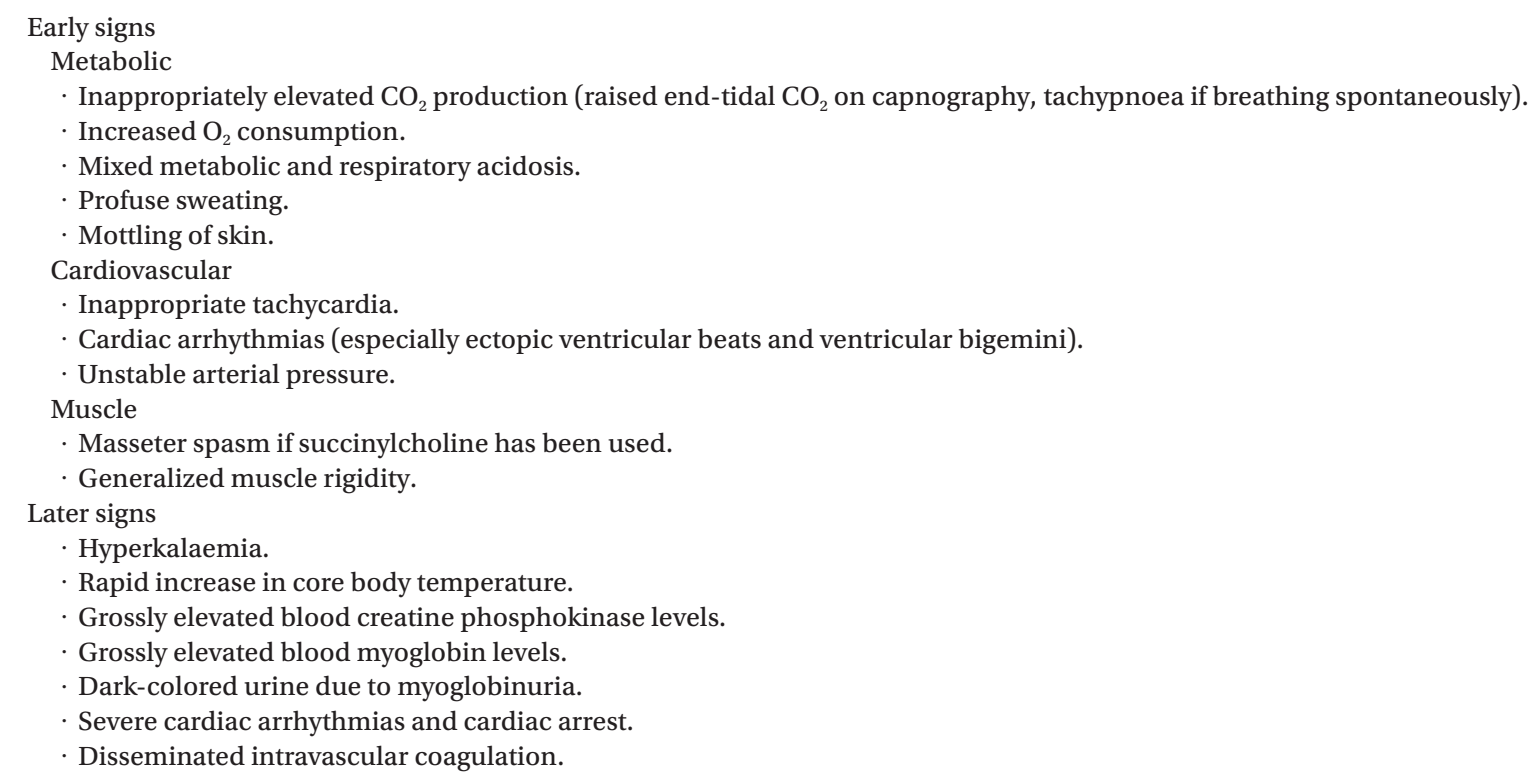


overcome and clinical signs subside. In some cases, the dose may be as much as $20 \mathrm{mg} / \mathrm{kg}$. If more than $20 \mathrm{mg} / \mathrm{kg}$ of dantrolene is not successful, the diagnosis of $\mathrm{MH}$ should be reconsidered. After the acute crisis has been controlled, dantrolene $1 \mathrm{mg} / \mathrm{kg}$ every 4 to 6 hours or alternatively $0.25 \mathrm{mg} / \mathrm{kg} / \mathrm{hr}$ continuous infusion for 24 hours is recommended. The prognosis is markedly influenced by the time between the onset of symptoms and the administration of dantrolene at a starting dose of $2.5 \mathrm{mg} / \mathrm{kg}[2,70]$. Although dantrolene sodium has no direct effect on actin/myosin binding, and no effect on the neuromuscular junction [73], dantrolene sodium may cause significant muscle weakness in patients with pre-existing neuromuscular disease [7]. Therefore, it should be used with extreme caution in those patients. Adverse effects, associated with dantrolene sodium, include muscle weakness, a sense of respiratory inadequacy, fatigue, dizziness, blurred vision, nausea, and thrombophlebitis [71].

Hyperthermia, a late clinical sign, should be treated with a variety of cooling techniques, including the use of cold $0.9 \%$ sodium chloride intravenous fluids, lavage of the stomach, bladder, rectal, or open cavity, and placement of ice packs on the neck, axilla, and groin. During the application of the cooling maneuver, core temperature should be monitored at the appropriate monitoring sites, including distal esophagus, nasopharynx, tympanic membrane, rectum, bladder, or axilla, as the patient should be cooled to no less than $38^{\circ} \mathrm{C}$ to avoid any additional problems.

Hyperkalemia may be life-threatening and precipitate cardiac arrhythmias; therefore, hyperkalemia should be aggressively treated with insulin, dextrose, sodium bicarbonate, and calcium.

The mixed respiratory and metabolic acidosis should be treated with hyperventilation at 2 to 3 times the predicted minute ventilation with $100 \%$ oxygen, and intravenous administration of $1-4 \mathrm{mEq} / \mathrm{kg}$ sodium bicarbonate. Repeated administration of sodium bicarbonate might be necessary because of a continuous efflux of lactate from cells as lactate only crosses through cell membranes slowly.

Initial management of cardiac arrhythmias includes treatment of the underlying acidosis and hyperkalemia as most arrhythmias during MH episode usually occur due to hyperkalemia and acidosis. If arrhythmias do not respond to the initial management, antiarrhythmic therapy using lidocaine, amiodarone and $\beta$-adrenoceptor blocking agents should be initiated. For the control of cardiac arrhythmias, calcium channel blocker (e.g., verapamil, diltiazem) should not be used as these drugs interact with dantrolene, and can lead to lifethreatening hyperkalemia, myocardial depression and ultimately cardiac arrest $[7,72]$.

As myoglobinemia and myoglobinuria, due to acute rhabdomyolysis, increase the risk of kidney damage, urine output should be maintained at greater than $2 \mathrm{ml} / \mathrm{kg} / \mathrm{hr}$ with more aggressive hydration, urine alkalinization, and diuretics [74].

In addition to the monitoring devices already in place (temperature probe, electrocardiogram, and arterial oxygen saturation), additional monitoring devices, such as an arterial cannula, a central venous catheter, as well as a urinary catheter in order to monitor renal function, should be used.

Laboratory testing should be performed immediately at the onset of an episode and periodically thereafter. Laboratory monitoring parameters include arterial and venous blood gases, electrolytes, coagulation profile, myoglobin and creatine kinase (CK). Arterial blood gases, serum electrolytes, and blood sugar should be checked every 15 minutes until the clinical signs of MH subside. The concentration of CK should be measured every 6-8 hours, until the value is normalized as CK is a guide to the status of muscle destruction, and will peak at 14-20 hours after muscle damage occurs [41].

After stabilization, the patient should be closely observed in ICU for at least $36-48$ hours because MH recurs in about $25 \%$ of patients within 48 hours of a treated episode $[7,75]$. Dantrolene sodium at a dose of $1 \mathrm{mg} / \mathrm{kg}$ should be continued every 4 to 6 hours intravenously during this period, depending on the clinical and laboratory parameters.

\section{MHS and Association with Idiopathic HyperCKemia and Other Neuromuscular Disorders}

\section{Idiopathic hyperCKemia}

Idiopathic hyperCKemia ( $\mathrm{IH}$ ) is a persistently elevated serum concentration of creatine kinase (CK) i.e., at least 3 serum CK levels more than twice normal over at least 3 months, without weakness or other significant neuromuscular symptoms [76,77]. Persistently elevated serum concentration of creatine kinase (CK) usually accompanies muscle weakness in patients with myopathies. However, it may also be found in individuals with a normal neurological examination, possibly due to subclinical or preclinical neuromuscular disorders, dystrophinopathy carrier state, hypothyroidism, hypoparathyroidism, alcoholism, or intake of statins and other drugs, and this condition is labeled asymptomatic hyperCKemia [78-80]. When the cause is not found, even after extensive investigations, the condition is defined as IH. Capasso et al. [80] studied a series of individuals with $\mathrm{IH}$ and found $\mathrm{IH}$ is familial in $46 \%$ of cases and familial $\mathrm{IH}$ is a benign genetically heterogeneous condition that is autosomal-dominant in at least $60 \%$ of cases, with higher penetrance in men.

Individuals with IH have long been suspected to have MHS and various studies have concluded $\mathrm{IH}$ to be a weak predictor 
of MHS [81-83]. Weglinski et al. [84] studied 49 asymptomatic individuals with $\mathrm{IH}$ and reported 24/40 (49\%), while in a more recent investigation of 37 Italian subjects with $\mathrm{IH}$, muscle contracture test detected one out of 37 individuals to be MHS (2.7\%) [85]. This discrepancy, between the results of the investigation, may be caused by different inclusion criteria of the investigation [35].

Although increased serum levels of $\mathrm{CK}$ are a valid reason to screen for underlying neuromuscular disorders, and to suspect MHS [86], no certain correlation has been reported between serum levels of CK and MHS [79,85,86]. Meanwhile, if serum level of CK is increased in an individual who has a first-degree relative proven MHS, then the individual can be considered to be susceptible [87]. Therefore, individuals with IH, who belonged to a family with a history of $\mathrm{MH}$, could be susceptible to $\mathrm{MH}$ but IH itself can be used as a predictor for MHS.

\section{Neuromuscular disorders associated with MHS}

Although individuals with certain neuromuscular disorders have long been suspected to have MHS, the risk of $\mathrm{MH}$ or $\mathrm{MH}-$ like reactions in patients with known neuromuscular disorders is not easy to determine. Because a number of case reports and small series have discussed patients with these disorders, who developed one or more of the following clinical symptoms: rigidity, increased temperature, arrhythmia, rhabdomyolysis, and hyperkalemia, during or after anesthesia, the anesthesiologist is confronted with the question of whether there is a true association between these disorders and MHS. However, it is important to recognize an association between these disorders and MHS, as the different underlying pathophysiological mechanism is to require different treatment and have different implications for future anesthetic management of the patient and their family.

The neuromuscular disorders that have a strong evidence for close association with MHS include central core disease (CCD), multi-minicore disease (MmCD) with RYR1 mutation and King Denborough syndrome [88-90].

Central Core Disease (CCD) is a rare hereditary myopathy with autosomal dominant inheritance, which is characterized clinically by muscle weakness of variable degree and histological by a predominance of type I fibers, containing clearly defined areas (central cores) lacking oxidative enzyme activity [91]. Individuals with CCD have been suspected to have MHS early, because individuals with MHS may have central cores on muscle biopsy [89], and individuals with CCD may be prone to $\mathrm{MH}$ episodes $[92,93]$. In the majority of cases, mutations associated with CCD are found mainly in RYR1 gene [26,94]. Among the reported mutations in RYR1 associated with MHS or CCD, more than 150 mutations are associated with MHS, approximately 100 mutations predispose to CCD, and more than 20 mutations appear to be associated with both MHS and CCD $[1,64]$. Therefore, Individuals with CCD should be considered as MHS and should be avoided triggering agents.

Multi-minicore disease (MmCD) is a recessively inherited congenital myopathy, characterized clinically by neonatal hypotonia, delayed motor development, and axial muscle weakness, which leads to the development of scoliosis and significant respiratory involvement, and histological by multifocal, wellcircumscribed areas (multi cores) with reduction of oxidative staining and low myofibrillar ATPase [95]. It is generally accepted that MmCD is associated with mutations in two genes, SEPN1 and RYR1 [96,97]. Even though the association with MHS is not well documented, clinical MH episodes have been recognized in few cases with $\mathrm{MmCD}[98,99]$.

King Denborough syndrome is a rare congenital myopathy associated with MHS, characterized by skeletal abnormalities, such as palmar simian line, pectus excavatum, winging of the scapulae, lumbar lordosis and mild thoracic scoliosis and dysmorphic features with characteristic facial appearance, such as dysmorphic facies, ptosis, down-slanting palpebral fissures, hypertelorism, epicanthic folds, low-set ears, malar hypoplasia, and micrognathia [7,100]. Inheritance of King-Denborough syndrome is unclear. Approximately one half of individuals with King-Denborough syndrome demonstrate the baseline elevated serum CK levels [101]. Recently, RYR1 mutation associated with King-Denborough syndrome are identified and reported [102]. Individuals with King-Denborough syndrome also should be considered as MHS and should not receive triggering agents.

\section{Current Status of Malignant Hyperthermia in Korea}

In recent years, $R Y R 1$ mutations related MHS have been identified and reported in Korea [99,103,104]. However, the current state of $\mathrm{MH}$ in Korea is far from optimistic, in terms of underestimation of the incidence, poor diagnostic tests for MHS and limited availability of dantrolene sodium.

Because there is no national based $\mathrm{MH}$ database and case reports published on journals have been the exclusive resource for estimating the incidence of $\mathrm{MH}$, the incidence of $\mathrm{MH}$ has long been underestimated in Korea. Only 27 cases have been reported during the year 1971-2007 [105]. The estimation of incidence based on the reported cases is underestimated, since the case reports published on journals only represent a part of the real number of $\mathrm{MH}$ cases. To estimate incidence with confidence, we should try to establish a national based $\mathrm{MH}$ database.

Although RYR1 mutations related MHS are discovered and reported in Korea, patients with MHS are mainly diagnosed on 
the basis of clinical manifestations without specific laboratory tests to confirm MHS; since there is no laboratory facility to confirm MHS who experienced MH episode during anesthesia.

Lastly, dantrolene sodium, the only specific drug for $\mathrm{MH}$ episode, can be available in Korea since several years ago. However, dantrolene sodium is still limitedly available, because dantrolene sodium is only supplied by KODC (Korean Orphan Drug Center).

The systemic efforts to improve the situations described above are required for the patients' safety in Korea.

\section{References}

1. Hirshey Dirksen SJ, Larach MG, Rosenberg H, Brandom BW, Parness J, Lang RS, et al. Future directions in malignant hyperthermia research and patient care. Anesth Analg 2011; 113: 1108-19.

2. Wappler F. Malignant hyperthermia. Eur J Anaesthesiol 2001; 18: 632-52.

3. Monnier N, Krivosic-Horber R, Payen JF, Kozak-Ribbens G, Nivoche $\mathrm{Y}$, Adnet $\mathrm{P}$, et al. Presence of two different genetic traits in malignant hyperthermia families: implication for genetic analysis, diagnosis, and incidence of malignant hyperthermia susceptibility. Anesthesiology 2002; 97: 1067-74.

4. Bachand M, Vachon N, Boisvert M, Mayer FM, Chartrand D. Clinical reassessment of malignant hyperthermia in Abitibi-Temiscamingue. Can J Anaesth 1997; 44: 696-701.

5. Wappler F. Anesthesia for patients with a history of malignant hyperthermia. Curr Opin Anaesthesiol 2010; 23: 417-22.

6. Britt BA, Kalow W. Malignant hyperthermia: a statistical review. Can Anaesth Soc J 1970; 17: 293-315.

7. Rosenberg H, Davis M, James D, Pollock N, Stowell K. Malignant hyperthermia. Orphanet J Rare Dis 2007; 2: 21.

8. Larach MG, Gronert GA, Allen GC, Brandom BW, Lehman EB. Clinical presentation, treatment, and complications of malignant hyperthermia in North America from 1987 to 2006. Anesth Analg 2010; 110: 498-507.

9. Rosero EB, Adesanya AO, Timaran CH, Joshi GP. Trends and outcomes of malignant hyperthermia in the United States, 2000 to 2005. Anesthesiology 2009; 110: 89-94.

10. Chamley D, Pollock NA, Stowell KM, Brown RL. Malignant hyperthermia in infancy and identification of a novel RYR1 mutation. Br J Anaesth 2000; 84: 500-4.

11. Ording H. Investigation of malignant hyperthermia susceptibility in Denmark. Dan Med Bull 1996; 43: 111-25.

12. Robinson RL, Curran JL, Ellis FR, Halsall PJ, Hall WJ, Hopkins PM, et al. Multiple interacting gene products may influence susceptibility to malignant hyperthermia. Ann Human Genet 2000; 64: 307-20.

13. Hernandez JF, Secrest JA, Hill L, McClarty SJ. Scientific advances in the genetic understanding and diagnosis of malignant hyperthermia. J Perianesth Nurs 2009; 24: 19-31.

14. Brady JE, Sun LS, Rosenberg H, Li G. Prevalence of malignant hyperthermia due to anesthesia in New York State, 2001-2005. Anesth Analg 2009; 109: 1162-6.

15. Iaizzo PA, Klein W, Lehmann-Horn F. Fura-2 detected myoplasmic calcium and its correlation with contracture force in skeletal muscle from normal and malignant hyperthermia susceptible pigs. Pflugers Arch 1988; 411: 648-53.

16. Struk A, Lehmann-Horn F, Melzer W. Voltage-dependent calcium release in human malignant hyperthermia muscle fibers. Biophys J 1998; 75: 2402-10.

17. Carrier L, Villaz M, Dupont Y. Abnormal rapid $\mathrm{Ca}^{2+}$ release from sarcoplasmic reticulum of malignant hyperthermia susceptible pigs. Biochim Biophys Acta 1991; 1064: 175-83.

18. Yang T, Allen PD, Pessah IN, Lopez JR. Enhanced excitationcoupled calcium entry in myotubes is associated with expression of RyR1 malignant hyperthermia mutations. J Biol Chem 2007; 282: 37471-8.

19. Nelson TE, Sweo T. $\mathrm{Ca}^{2+}$ uptake and $\mathrm{Ca}^{2+}$ release by skeletal muscle sarcoplasmic reticulum: differing sensitivity to inhalational anesthetics. Anesthesiology 1988; 69: 571-7.

20. Lai FA, Meissner G. The muscle ryanodine receptor and its intrinsic $\mathrm{Ca}^{2+}$ channel activity. J Bioenerg Biomembr 1989; 21: 227-46.

21. Kim DH, Sreter FA, Ikemoto N. Involvement of the $60 \mathrm{kDa}$ phosphoprotein in the regulation of $\mathrm{Ca}^{2+}$ release from sarcoplasmic reticulum of normal and malignant hyperthermia susceptible pig muscles. Biochim Biophys Acta 1988; 945: 246-52.

22. Fill M, Coronado R, Mickelson JR, Vilven J, Ma JJ, Jacobson BA, et al. Abnormal ryanodine receptor channels in malignant hyperthermia. Biophys J 1990; 57: 471-5.

23. Nelson TE, Lin M, Volpe P. Evidence for intraluminal $\mathrm{Ca}^{++}$regulatory site defect in sarcoplasmic reticulum from malignant hyperthermia pig muscle. J Pharmacol Exp Ther 1991; 256: 645-9.

24. McCarthy TV, Quane KA, Lynch PJ. Ryanodine receptor mutations in malignant hyperthermia and central core disease. Hum Mutat 2000; 15: 410-7.

25. Maclennan DH, Zvaritch E. Mechanistic models for muscle diseases and disorders originating in the sarcoplasmic reticulum. Biochim Biophys Acta 2011; 1813: 948-64.

26. Robinson R, Carpenter D, Shaw MA, Halsall J, Hopkins P. Mutations in RYR1 in malignant hyperthermia and central core disease. Hum Mutat 2006; 27: 977-89.

27. Sambuughin N, Holley H, Muldoon S, Brandom BW, de Bantel AM, Tobin JR, et al. Screening of the entire ryanodine receptor type 1 coding region for sequence variants associated with malignant hyperthermia susceptibility in the north american population. Anesthesiology 2005; 102: 515-21.

28. Brandom BW. Genetics of malignant hyperthermia. Sci World J 2006; 6: 1722-30.

29. Monnier N, Procaccio V, Stieglitz P, Lunardi J. Malignant-hyperthermia susceptibility is associated with a mutation of the alpha 1-subunit of the human dihydropyridine-sensitive l-type voltagedependent calcium-channel receptor in skeletal muscle. Am J Hum Genet 1997; 60: 1316-25.

30. Marchant CL, Ellis FR, Halsall PJ, Hopkins PM, Robinson RL. Mutation analysis of two patients with hypokalemic periodic paralysis and suspected malignant hyperthermia. Muscle Nerve 2004; 30 114-7.

31. Hopkins PM. Malignant hyperthermia: advances in clinical management and diagnosis. Br J Anaesth 2000; 85: 118-28.

32. Litman RS, Rosenberg H. Malignant hyperthermia: update on 
susceptibility testing. JAMA 2005; 293: 2918-24.

33. Iaizzo PA, Kehler CH, Carr RJ, Sessler DI, Belani KG. Prior hypothermia attenuates malignant hyperthermia in susceptible swine. Anesth Analg 1996; 82: 803-9.

34. Levitt RC, Nouri N, Jedlicka AE, McKusick VA, Marks AR, Shutack JG, et al. Evidence for genetic heterogeneity in malignant hyperthermia susceptibility. Genomics 1991; 11: 543-7.

35. Bandschapp O, Girard T. Malignant hyperthermia. Swiss Med Wkly 2012; 142: w13652.

36. Heffron JJ. Malignant hyperthermia: biochemical aspects of the acute episode. Br J Anaesth 1988; 60: 274-8.

37. Kim DC, Lim HR, Han YJ. Early recognition of malignant hyperthermia with capnography: A case report. Korean J Anesthesiol 2002; 43: 667-72.

38. Tautz TJ, Urwyler A, Antognini JF, Riou B. Case scenario: Increased end-tidal carbon dioxide: a diagnostic dilemma. Anesthesiology 2010; 112: 440-6.

39. Karan SM, Crowl F, Muldoon SM. Malignant hyperthermia masked by capnographic monitoring. Anesth Analg 1994; 78: 590-2.

40. Denborough MA. Anaesthetic deaths in a family (letter). Lancet 1960; 2: 45.

41. Ali SZ, Taguchi A, Rosenberg H. Malignant hyperthermia. Best Pract Res Clin Anaesthesiol 2003; 17: 519-33.

42. Nelson TE. Porcine malignant hyperthermia: critical temperatures for in vivo and in vitro responses. Anesthesiology 1990; 73: 449-54.

43. Sessler DI. Temperature monitoring and perioperative thermoregulation. Anesthesiology 2008; 109: 318-38.

44. Stratman RC, Flynn JD, Hatton KW. Malignant hyperthermia: a pharmacogenetic disorder. Orthopedics 2009; 32: 835.

45. Larach MG, Brandom BW, Allen GC, Gronert GA, Lehman EB. Cardiac arrests and deaths associated with malignant hyperthermia in North America from 1987 to 2006: a report from the north american malignant hyperthermia registry of the malignant hyperthermia association of the United States. Anesthesiology 2008; 108: 603-11.

46. Larach MG, Localio AR, Allen GC, Denborough MA, Ellis FR, Gronert GA, et al. A clinical grading scale to predict malignant hyperthermia susceptibility. Anesthesiology 1994; 80: 771-9.

47. Von Richthofen V, Wappler F, Scholz J, Fiege M, Schulte am Esch J. Evaluation of malignant hyperthermia episodes with the Clinical Grading Scale. Anasthesiol Intensivmed Notfallmed Schmerzther 1998; 33: 244-9.

48. Lee JW, Moon SS, Lee JR, Kim DC. Reevaluation by clinical grading scale for malignant hyperthermia reported in Korean Journal of Anesthesiology. Korean J Anesthesiol 2008; 54: 640-5.

49. Larach MG, MacLennan DH. How carefully can we phenotype patients suspected of malignant hyperthermia susceptibility? Anesthesiology 1999; 90: 645-8.

50. Kalow W, Britt BA, Terreau ME, Haist C. Metabolic error of muscle metabolism after recovery from malignant hyperthermia. Lancet 1970; 2: 895-8.

51. Ellis FR, Harriman DG, Keaney NP, Kyei-Mensah K, Tyrrell JH. Halothane-induced muscle contracture as a cause of hyperpyrexia. Br J Anaesth 1971; 43: 721-2.

52. A protocol for the investigation of malignant hyperpyrexia (MH) susceptibility. The Malignant Hyperpyrexia Group. Br J Anaesth
1984; 56: 1267-9.

53. Larach MG. Standardization of the caffeine halothane muscle contracture test. North American Malignant Hyperthermia Group. Anesth Analg 1989; 69: 511-5.

54. Fletcher JE, Conti PA, Rosenberg H. Comparison of North American and European malignant hyperthermia group halothane contracture testing protocols in swine. Acta Anaesthesiol Scand 1991; 35: 483-7.

55. Allen GC, Larach MG, Kunselman AR. The sensitivity and specificity of the caffeine-halothane contracture test: a report from the North American Malignant Hyperthermia Registry. The North American Malignant Hyperthermia Registry of MHAUS. Anesthesiology 1998; 88: 579-88.

56. Ording H, Brancadoro V, Cozzolino S, Ellis FR, Glauber V, Gonano $\mathrm{EF}$, et al. In vitro contracture test for diagnosis of malignant hyperthermia following the protocol of the European MH Group: results of testing patients surviving fulminant $\mathrm{MH}$ and unrelated low-risk subjects. The European Malignant Hyperthermia Group. Acta Anaesthesiol Scand 1997; 41: 955-66.

57. Fletcher JE,, Rosenburg H, Aggarwal M. Comparison of European and North American malignant hyperthermia diagnostic protocol outcomes for use in genetic studies. Anesthesiology 1999; 90: 654-61.

58. Ording H, Bendixen D. Sources of variability in halothane and caffeine contracture tests for susceptibility to malignant hyperthermia. Eur J Anaesthesiol 1992; 9: 367-76.

59. Nelson TE, Rosenberg H, Muldoon SM. Genetic testing for malignant hyperthermia in North America. Anesthesiology 2004; 100: 212-4.

60. Urwyler A, Deufel T, McCarthy T, West S. Guidelines for molecular genetic detection of susceptibility to malignant hyperthermia. Br J Anaesth 2001; 86: 283-7.

61. Rosenberg H, Rueffert H. Clinical utility gene card for: malignant hyperthermia. Eur J Hum Genet 2011;19.

62. Girard T, Treves S, Voronkov E, Siegemund M, Urwyler A. Molecular genetic testing for malignant hyperthermia susceptibility. Anesthesiology 2004; 100: 1076-80.

63. McCarthy TV, Healy JM, Heffron JJ, Lehane M, Deufel T, LehmannHorn F, et al. Localization of the malignant hyperthermia susceptibility locus to human chromosome 19q12-13.2. Nature 1990; 343: 562-4.

64. Rosenberg H, Sambuughin N, Dirksen R. Malignant hyperthermia susceptibility. In: GeneReviews at GeneTests: Medical Genetics Information Resource database online. Seattle: University of Washington, 1997-2011. Updated January 19, 2010. Available at http://www.genetests.org. [Accessed August 1, 2012].

65. Deufel T, Sudbrak R, Feist Y, Rübsam B, Du Chesne I, Schäfer KL, et al. Discordance, in a malignant hyperthermia pedigree, between in vitro contracture-test phenotypes and haplotypes for the MHS1 region on chromosome 19q12-13.2, comprising the C1840T transition in the RYR1 gene. Am J Hum Genet 1995; 56: 1334-42.

66. MacLennan DH. Discordance between phenotype and genotype in malignant hyperthermia. Curr Opin Neurol 1995; 8: 397-401.

67. Robinson R, Hopkins P, Carsana A, Gilly H, Halsall J, Heytens L, et al. Several interacting genes influence the malignant hyperthermia phenotype. Hum Genet 2003; 112: 217-8.

68. Robinson RL, Anetseder MJ, Brancadoro V, van Broekhoven C, 
Carsana A, Censier K, et al. Recent advances in the diagnosis of malignant hyperthermia susceptibility: how confident can we be of genetic testing? Eur J Hum Genet 2003; 11: 342-8.

69. Robinson RL, Anetseder MJ, Brancadoro V, van Broekhoven C, Carsana A, Censier K, et al. Recent advances in the diagnosis of malignant hyperthermia susceptibility: how confident can we be of genetic testing? Eur J Hum Genet 2003; 11: 342-8.

70. Glahn KP, Ellis FR, Halsall PJ, Müller CR, Snoeck MM, Urwyler A, et al. Recognizing and managing a malignant hyperthermia crisis: guidelines from the European Malignant Hyperthermia Group. Br J Anaesth 2010; 105: 417-20.

71. Inan S, Wei H. The cytoprotective effects of dantrolene: a ryanodine receptor antagonist. Anesth Analg 2010; 111: 1400-10.

72. Krause T, Gerbershagen MU, Fiege M, Weisshorn R, Wappler F. Dantrolene--a review of its pharmacology, therapeutic use and new developments. Anaesthesia 2004; 59: 364-73.

73. Kurihara T, Brooks JE. Excitation-contraction uncoupling. The effect of hyperosomolar glycerol solution and dantrolene sodium on mammalian muscle in vitro. Arch Neurol 1975; 32: 92-7.

74. Bosch X, Poch E, Grau JM. Rhabdomyolysis and acute kidney injury. N Engl J Med 2009; 361: 62-72.

75. Burkman JM, Posner KL, Domino KB. Analysis of the clinical variables associated with recrudescence after malignant hyperthermia reactions. Anesthesiology 2007; 106: 901-6.

76. Afifi AK. Idiopathic hyperCKemia revisited. J Child Neurol 1998; 13: 251-2.

77. Sunohara N, Takagi A, Nonaka I, Sugita H, Satoyoshi E. Idiopathic hyperCKemia. Neurology 1984; 34: 544-7.

78. Prelle A, Tancredi L, Sciacco M, Chiveri L, Comi GP, Battistel A, et al. Retrospective study of a large population of patients with asymptomatic or minimally symptomatic raised serum creatine kinase levels. J Neurol 2002; 249: 305-11.

79. Simmons Z, Peterlin BL, Boyer PJ, Towfighi J. Muscle biopsy in the evaluation of patients with modestly elevated creatine kinase levels. Muscle Nerve 2003; 27: 242-4.

80. Capasso M, De Angelis MV, Di Muzio A, Scarciolla O, Pace M, Stuppia L, et al. Familial idiopathic hyper-CKemia: an underrecognized condition. Muscle Nerve 2006; 33: 760-5.

81. Ellis FR, Clarke IM, Modgill M, Currie S, Harriman DG. Evaluation of creatinine phosphokinase in screening patients for malignant hyperpyrexia. Br Med J 1975; 3: 511-3.

82. Paasuke RT, Brownell AK. Serum creatine kinase level as a screening test for susceptibility to malignant hyperthermia. JAMA 1986; 255 : 769-71.

83. Lingaraju N, Rosenberg H. Unexplained increases in serum creatine kinase levels: its relation to malignant hyperthermia susceptibility. Anesth Analg 1991; 72: 702-5.

84. Weglinski MR, Wedel DJ, Engel AG. Malignant hyperthermia testing in patients with persistently increased serum creatine kinase levels. Anesth Analg 1997; 84: 1038-41.

85. Malandrini A, Orrico A, Gaudiano C, Gambelli S, Galli L, Berti G, et al. Muscle biopsy and in vitro contracture test in subjects with idiopathic hyperCKemia. Anesthesiology 2008; 109: 625-8.

86. Kasi PM. Malignant Hyperthermia and Idiopathic HyperCKemia. Case Report Med 2011; 2011: 194296.

87. Monnier N, Kozak-Ribbens G, Krivosic-Horber R, Nivoche Y, Qi D,
Kraev N, et al. Correlations between genotype and pharmacological, histological, functional, and clinical phenotypes in malignant hyperthermia susceptibility. Hum Mutat 2005; 26: 413-25.

88. Davis PJ, Brandom BW. The association of malignant hyperthermia and unusual disease: when you're hot you're hot, or maybe not. Anesth Analg 2009; 109: 1001-3.

89. Klingler W, Rueffert H, Lehmann-Horn F, Girard T, Hopkins PM. Core myopathies and risk of malignant hyperthermia. Anesth Analg 2009; 109: 1167-73.

90. Benca J, Hogan K. Malignant hyperthermia, coexisting disorders, and enzymopathies: risks and management options. Anesth Analg 2009; 109: 1049-53.

91. Quinlivan RM, Muller CR, Davis M, Laing NG, Evans GA, Dwyer J, et al. Central core disease: clinical, pathological, and genetic features. Arch Dis Child 2003; 88: 1051-5.

92. Frank JP, Harati Y, Butler IJ, Nelson TE, Scott CI. Central core disease and malignant hyperthermia syndrome. Ann Neurol 1980; 7 : 11-7.

93. Jungbluth H. Central core disease. Orpha J Rare Dis 2007; 2: 25.

94. Wu S, Ibarra MC, Malicdan MC, Murayama K, Ichihara Y, Kikuchi $\mathrm{H}$, et al. Central core disease is due to RYR1 mutations in more than 90\% of patients. Brain 2006; 129: 1470-80.

95. Jungbluth H. Multi-minicore disease. Orpha J Rare Dis 2007; 2: 31.

96. Jungbluth H, Zhou H, Hartley L, Halliger-Keller B, Messina S, Longman C, et al. Minicore myopathy with ophthalmoplegia caused by mutations in the ryanodine receptor type 1 gene. Neurology 2005; 65: 1930-5.

97. Ferreiro A, Quijano-Roy S, Pichereau C, Moghadaszadeh B, Goemans N, Bonnemann C, et al. Mutations of the selenoprotein N gene, which is implicated in rigid spine muscular dystrophy, cause the classical phenotype of multiminicore disease: reassessing the nosology of early-onset myopathies. Am J Hum Genet 2002; 71: 73949 .

98. Koch BM, Bertorini TE, Eng GD, Boehm R. Severe multicore disease associated with reaction to anaesthesia. Arch Neurol 1985; 42: 1204-6.

99. Jeong SK, Kim DC, Cho YG, Sunwoo IN, Kim DS. A double mutation of the ryanodine receptor type 1 gene in a malignant hyperthermia family with multiminicore myopathy. J Clin Neurol 2008; 4: 123-30.

100. King JO, Denborough MA. Anaesthetic-induced malignant hyperpyrexia in children. J Pediatr 1973; 83: 37-40.

101. Isaacs H, Badenhorst ME. Dominantly inherited malignant hyperthermia (MH) in the King-Denborough syndrome. Muscle Nerve 1992; 15: 740-2.

102. D'Arcy CE, Bjorksten A, Yiu EM, Bankier A, Gillies R, McLean CA, et al. King-Denborough syndrome caused by a novel mutation in the ryanodine receptor gene. Neurology 2008; 71: 776-7.

103. Kim DC, Kim DS. Identification of G7304A mutation in the ryanodine receptor type 1 gene in a patient with malignant hyperthermia and an extended pedigree study in a korean malignant hyperthermia family. Korean J Anesthesiol 2003; 44: 56-64.

104. Lee H, Kim DC, Lee JH, Cho YG, Lee HS, Choi SI, et al. Molecular genetic analysis of the ryanodine receptor gene (RYR1) in Korean malignant hyperthermia families. Korean J Lab Med 2010; 30: 702-10.

105. Lee JW, Moon SS, Lee JR, Kim DC. Reevaluation by clinical grading scale for malignant hyperthermia reported in Korean journal of anesthesiology. Korean J Anesthesiol 2008; 54: 640-5. 